\title{
A case of recurrent infective endocarditis following colonoscopy
}

A 57-year-old man with a history of prosthetic valve replacement at age 47 was admitted to our hospital with bloody stools. A colonoscopy revealed a rectal polyp, eventually diagnosed as a tubulovillous adenoma measuring $30 \mathrm{~mm}$ in diameter (๑ Fig. 1).

Following a $10-\mathrm{mL}$ submucosal saline injection, the rectal polyp was removed by piecemeal endoscopic mucosal resection within 5 minutes. The day after the examination, the patient developed high grade fever (body temperature $39^{\circ} \mathrm{C}$ ). Blood cultures grew Enterococcus faecalis and transesophageal echocardiography showed vegetations on the mitral valve and tricuspid valve. The patient was diagnosed as having infective endocarditis and treated with ampicillin and gentamicin sulfate. After 6 weeks of antibiotic therapy, the patient was discharged without any complications. At 18 months, the patient underwent a follow-up colonoscopy and an ascending colon polyp measuring $4 \mathrm{~mm}$ in diameter was removed without antibiotic prophylaxis. In the evening of the day after the examination, the patient developed fever (body temperature $37^{\circ} \mathrm{C}$ ). Blood cultures grew $E$. faecalis and transesophageal echocardiography on the third day showed a vegetation on the tricuspid valve. Antibiotic therapy consisting of ampicillin and gentamicin sulfate was given for 4 weeks after which the patient was discharged without any complications.

The causes of endocarditis following colonoscopy remain unclear. Submucosal injection or piecemeal resection may be one of the risk factors for endocarditis. According to the American Heart Association 2007 guidelines [1] and the European Society of Cardiology 2009 guidelines [2], antibiotic prophylaxis is not recommended prior to gastrointestinal tract proce-

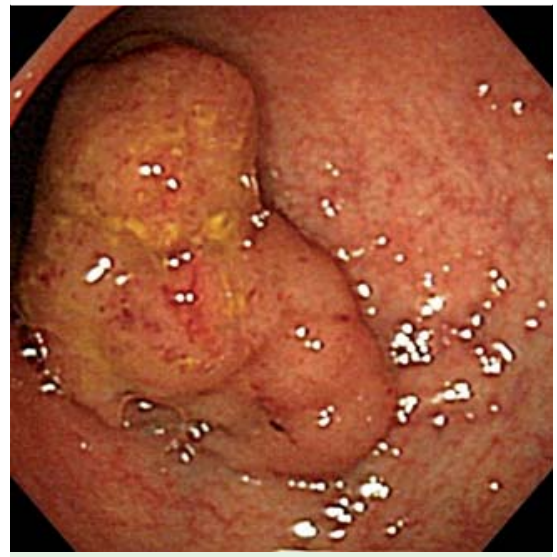

Fig. 1 Colonoscopy showing a rectal polyp measuring $30 \mathrm{~mm}$ in diameter and which turned out to be a tubulovillous adenoma.

dures. However, the guidelines may need to be reviewed as antibiotic prophylaxis is probably required in some patients, especially those with a history of infective endocarditis.

\section{Competing interests: None}

Endoscopy_UCTN_Code_CPL_1AJ_2AD

Y. Sekino ${ }^{1}$, N. Fujisawa ${ }^{2}$, K. Suzuki ${ }^{2}$,

K. Akimoto ${ }^{2}$, A. Takahata², K. Miharada², S. Koyama ${ }^{2}$, H. lida ${ }^{1}$, H. Endo ${ }^{1}$,

K. Hosono ${ }^{1}$, Y. Sakamoto ${ }^{1}$, H. Taka-

hashi $^{1}$, T. Koide ${ }^{1}$, C. Tokoro ${ }^{1}$, Y. Abe ${ }^{1}$,

S. Maeda ${ }^{1}$, A. Nakajima ${ }^{1}$, A. Tatsumoto ${ }^{3}$,

H. Sakurada ${ }^{3}$, M. Inamori ${ }^{1}$

1 Gastroenterology Division, Yokohama

City University Hospital, Japan

2 Department of Gastroenterology, Tokyo Metropolitan Hiroo Hospital, Japan

3 Department of Cardiology, Tokyo Metropolitan Hiroo Hospital, Japan
References

1 Quality of Care and Outcomes Research Interdisciplinary Working Group. Prevention of infective endocarditis. Circulation 2007; 116: 1736 - 1754

2 Task Force on the Prevention, Diagnosis, and Treatment of Infective Endocarditis of the European Society of Cardiology; European Society of Clinical Microbiology and Infectious Diseases; International Society of Chemotherapy for Infection and Cancer. Habib $G$, Hoen B, Tornos P et al. Guidelines on the prevention, diagnosis, and treatment of infective endocarditis (new version 2009): the Task Force on the Prevention, Diagnosis, and Treatment of Infective Endocarditis of the European Society of Cardiology (ESC). Eur Heart J 2009; 30: 2369-2413

Bibliography

DOI 10.1055/s-0030-1255719

Endoscopy 2010; 42: E217

(c) Georg Thieme Verlag KG Stuttgart · New York . ISSN 0013-726X

Corresponding author

M. Inamori, MD, PhD

Gastroenterology Division

Yokohama City University Hospital

3-9 Fukuura

Kanazawa-ku

Yokohama 236-0004

Japan

Fax: +81-45-784-3546

inamorim@med.yokohama-cu.ac.jp 